\title{
Co-ordinate Interleaved Spatial Multiplexing with Channel State Information
}

\author{
K. V. Srinivas, Student Member, IEEE, R. D. Koilpillai, Member, IEEE, Srikrishna Bhashyam, Member, IEEE, \\ and K. Giridhar, Member, IEEE
}

\begin{abstract}
Performance of spatial multiplexing multiple-input multiple-output (MIMO) wireless systems can be improved with channel state information (CSI) at both ends of the link. This paper proposes a new linear diagonal MIMO transceiver, referred to as co-ordinate interleaved spatial multiplexing (CISM). With CSI at transmitter and receiver, CISM diagonalizes the MIMO channel and interleaves the co-ordinates of the input symbols (from rotated QAM constellations) transmitted over different eigenmodes. The analytical and simulation results show that with co-ordinate interleaving across two eigenmodes, the diversity gain of the data stream transmitted over the weaker eigenmode becomes equal to that of the data transmitted on the stronger eigenmode, resulting in a significant improvement in the overall diversity. The diversity-multiplexing tradeoff (DMT) is analyzed for CISM and is shown that it achieves higher diversity gain at all positive multiplexing gains compared to existing diagonal transceivers. Over rank $n$ MIMO channels, with input symbols from rotated $n$-dimensional constellations, the DMT of CISM is a straight line connecting the endpoints $\left(0, N_{t} N_{r}\right)$ and $\left(\min \left\{N_{t}, N_{r}\right\}, 0\right)$, where $N_{t}$ and $N_{r}$ are the number of transmit and receive antennas, respectively.
\end{abstract}

Index Terms-MIMO, spatial multiplexing, diversity, beamforming, co-ordinate interleaving, precoding, diversitymultiplexing gain tradeoff.

\section{INTRODUCTION}

$\mathbf{M}$ ULTIPLE-INPUT multiple-output (MIMO) wireless systems employing multiple antennas at each end have the potential to improve data throughput over time varying fading channels [1]. In rich scattering environments, MIMO channels provide multiple paths for data transmission and offer multiple degrees of freedom to communicate [2]. Multiple paths can be exploited to obtain diversity gain by transmitting the same symbol over all the paths, and multiple degrees of freedom can be used to increase the data rate through spatial multiplexing [3].

Early spatial multiplexing systems were developed assuming channel state information (CSI) only at the receiver. With CSI at both ends, the transmit and receive algorithms can be jointly designed to pre-process the data in a channel-dependent way such that the system performance is further improved. This is commonly known as transceiver design/optimization

Manuscript received April 19, 2005; revised January 11, 2007; accepted October 23, 2008. The associate editor coordinating the review of this letter and approving it for publication was X.-G. Xia.

The authors are with the Department of Electrical Engineering, Indian Institute of Technology Madras, Chennai, india, 600036 (e-mail: \{kvsri, koilpillai,skrishna, giri\}@tenet.res.in).

This work was financially supported by Centre of Excellence in Wireless Technology (CEWiT), Chennai, India.

Part of this work was presented at IEEE Intl. Conf. Acoustics, Speech, and Signal Processing, (ICASSP) 2006, Toulouse, France.

Digital Object Identifier 10.1109/TWC.2009.071391 and many emerging wireless standards are actively considering such concepts.

Several optimality criteria have been used for designing MIMO transceivers with CSI at both ends. Some of them include, minimizing the sum of the mean square error (MSE) of all data streams under an average power constraint [4], minimizing the weighted sum of MSEs [5], minimizing the product of MSEs with a peak power constraint [6] and maximizing the minimum Euclidean distance between the received signal points [7]. All these linear transceivers diagonalize the MIMO channel as it simplifies the solution through scalarization. Optimality of diagonal MIMO structures has been investigated in [8], where the authors have proposed a unified framework for designing diagonal MIMO transceivers according to a variety of optimality criterion including minimizing the average (or, overall) error probability. The uniform channel decomposition (UCD) scheme of [9], referred as UCD-VBLAST, is a non-linear transceiver that decomposes the MIMO channel into multiple identical sub-channels and equalizes the MSEs of all the data streams.

Diagonalizing the MIMO channel through singular value decomposition (SVD) and transmitting multiple data streams over the resulting parallel eigen sub-channels or eigenmodes, is a well known and simple linear diagonal MIMO transceiver. It is referred to as SVD transceiver (SVDTR) in rest of the paper. The overall error rate performance of SVDTR, as well as the more advanced diagonal transceivers such as those proposed in [8], is degraded by the lower diversity gains of weaker eigenmodes [10]. Most of the sophisticated diagonal transceivers proposed in the literature improve coding gain (compared to SVDTR) but not the diversity gain. Often, to improve the diversity gain, data is transmitted only on the stronger eigenmodes but this would render the remaining degrees of freedom un-used.

In this paper, we propose a new linear diagonal transceiver, referred to as co-ordinate interleaved spatial multiplexing (CISM) that improves the diversity gain of weaker eigenmodes. CISM diagonalizes the MIMO channel through SVD and interleaves the co-ordinates of the symbols (chosen from rotated QAM constellations) transmitted over strong and weak eigenmodes. Analytical results presented in the paper show that co-ordinate interleaving across eigenmodes $k$ and $l$, having diversity gains $g_{d}(k)$ and $g_{d}(l)$, respectively, results in a diversity gain of $\max \left\{g_{d}(k), g_{d}(l)\right\}$ on both the channels. Thus, CISM significantly improves overall error rate performance without necessarily leaving out the weak eigenmodes. Further, the diversity-multiplexing tradeoff is analyzed for CISM to show that it achieves higher diversity gain at all multiplexing 
gains compared to existing diagonal transceivers.

Notation: Bold upper case and lower case letters denote matrices and vectors, respectively. $a_{k l}$ denotes $(k, l)^{t h}$ entry of matrix $\mathbf{A}$ and $b_{k}$ denotes $k^{\text {th }}$ element in vector $\mathbf{b}$. $\Re x$ and $\Im x$ are real and imaginary parts of $x$ and $\jmath:=\sqrt{-1} . \mathbf{I}_{N},(\cdot)^{T}$ and $(\cdot)^{H}$ denotes $N \times N$ identity matrix, transposition and conjugate transposition, respectively. $\operatorname{Pr}\{\cdot\}, E[\cdot]$ and $\|\cdot\|$ denote the probability of the event in brackets, expectation and $L_{2}$-norm of a vector, respectively. $\lfloor a\rfloor$ indicates largest integer less than or equal to $a$.

\section{System Model}

Consider a $N_{t} \times N_{r}$ MIMO system with $N_{t}$ transmit and $N_{r}$ receive antennas. The discrete time baseband input-output relation of the MIMO channel is

$$
\mathbf{y}=\mathbf{H s}+\mathbf{n},
$$

where $\mathbf{s} \in \mathbb{C}^{N_{t} \times 1}$ and $\mathbf{y} \in \mathbb{C}^{N_{r} \times 1}$ are transmit and receive symbol vectors, respectively, $\mathbf{H} \in \mathbb{C}^{N_{r} \times N_{t}}$ is the channel matrix, and $\mathbf{n} \in \mathbb{C}^{N_{r} \times 1}$ is the additive noise vector with $n_{i} \sim \mathcal{C N}\left(0, \sigma^{2}\right)$ and $E\left[\mathbf{n n}^{H}\right]=\sigma^{2} \mathbf{I}_{N_{r}}$. The channel gains $\left\{h_{i j}\right\}$ are assumed to be independent, frequency-flat Rayleigh fading and hence, $\left\{h_{i j}\right\}$ are i.i.d with $h_{i j} \sim \mathcal{C N}(0,1)$. We define $m:=\max \left\{N_{t}, N_{r}\right\}, n:=\min \left\{N_{t}, N_{r}\right\}$ and $\mathbf{W}:=$ $\mathbf{H H}^{H}$ when $N_{r} \leq N_{t}$ and $\mathbf{W}:=\mathbf{H}^{H} \mathbf{H}$ when $N_{r}>N_{t}$, and consider a slow-fading environment. For uncorrelated Rayleigh MIMO channels, $\operatorname{rank}(\mathbf{H})=n=\min \left\{N_{t}, N_{r}\right\}$.

Let the SVD of $\mathbf{H}$ be $\mathbf{H}=\mathbf{U} \boldsymbol{\Lambda} \mathbf{V}^{H}$, where $\mathbf{U} \in \mathbb{C}^{N_{r} \times N_{r}}$ and $\mathbf{V} \in \mathbb{C}^{N_{t} \times N_{t}}$ are unitary matrices, and $\boldsymbol{\Lambda} \in \mathbb{R}^{N_{r} \times N_{t}}$ is a diagonal matrix with $\sqrt{\lambda_{k}} \in \mathbb{R}^{+}$, the $k^{\text {th }}$ largest singular value of $\mathbf{H}$, as its $k^{\text {th }}$ diagonal element [11]. Let $\mathbf{x} \in \mathbb{C}^{K \times 1}, K \leq$ $n$, be the symbol vector with $x_{k} \in \mathcal{X}_{k}, 1 \leq k \leq K$, where $\mathcal{X}_{k}$ is a unit energy QAM signal set employed on the $k^{\text {th }}$ eigen sub-channel, and $E\left[\mathbf{x x}^{H}\right]=\mathbf{I}_{K}$. By transmitting $\mathbf{s}=\mathbf{V}_{K} \mathbf{P} \mathbf{x}$, where $\mathbf{V}_{K}$ contains the first $K$ columns of $\mathbf{V}$, and by premultiplying the received vector $\mathbf{y}$ with $\mathbf{U}_{K}^{H}$, we get

$$
\mathbf{r}=\boldsymbol{\Lambda}_{K} \mathbf{P} \mathbf{x}+\mathbf{w}
$$

where $\mathbf{r}=\mathbf{U}_{K}^{H} \mathbf{y}, \mathbf{w}=\mathbf{U}_{K}^{H} \mathbf{n}$ and $\boldsymbol{\Lambda}_{K}=\operatorname{diag}\left(\left\{\sqrt{\lambda_{k}}\right\}_{k=1}^{K}\right)$. $\mathbf{P}=\operatorname{diag}\left(\left\{\sqrt{p_{k}}\right\}_{k=1}^{K}\right)$ where $p_{k} \geq 0$ is the power allocated to the $k^{t h}$ data stream. The transmit power is constrained such that $\sum_{k=1}^{K} p_{k} \leq P$, and SNR $:=P / \sigma^{2}$ is the average SNR at each receive antenna.

$K$ denotes the number of active eigenmodes or eigen subchannels and the transmission scheme discussed above is referred to as SVD transceiver (SVDTR) in rest of the paper. It is also called multiple beamforming [12], and maximum eigenmode beamforming (MEBF) corresponds to $K=1$.

\section{A. Performance Measures: Diversity gain and Diversity- Multiplexing Tradeoff}

At high SNR, the average symbol error probability (SEP),

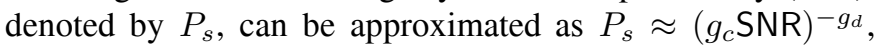
where $g_{c}$ is the coding gain and $g_{d}$ is referred to as the diversity gain [13]. The diversity gain $g_{d}(k)$ of $k^{\text {th }}$ eigen subchannel has been determined in [10] as

$$
g_{d}(k)=(m-k+1)(n-k+1), \quad k=1, \ldots, n
$$

and was also shown that $g_{d}(k)$ does not improve with spatial power allocation. With $K$ active eigenmodes, the overall diversity gain of SVDTR is $g_{d}=\min _{k=1, \ldots, K}\left\{g_{d}(k)\right\}$. Diversity gains of most of the advanced diagonal transceivers are same as that of SVDTR [10].

The diversity-multiplexing tradeoff (DMT) [2] is a more fundamental performance measure of a MIMO transceiver in slow-fading scenario as it captures diversity gain and multiplexing gain. A diversity gain $d(r)$ is achieved at a multiplexing gain $r$, if the data rate scales as $R=r \log \mathrm{SNR}$ $\mathrm{b} / \mathrm{s} / \mathrm{Hz}$ and the SEP is

$$
P_{s}(r \log \mathrm{SNR}) \doteq \mathrm{SNR}^{-d(r)}
$$

where $\doteq$ denotes exponential equality [2]. Note that $g_{d}$ is the diversity gain for fixed input data rate (i.e., $g_{d}=d(0)$ ), and to distiguish from $d(r)$, it is referred to as classical diversity gain [14] wherever necessary. The DMT for SVDTR has been evaluated in [12]. We derive the DMT of CISM in section V.

\section{Co-ordinate Interleaved Spatial Multiplexing}

In this section, co-ordinate interleaved spatial multiplexing (CISM) is described. The proposed scheme is based on coordinate interleaving $(\mathrm{CI})$, a technique which was originally proposed to exploit the co-ordinate, or, component level diversity for single antenna transmission over Rayleigh fading channels [15], [16]. The idea is to interleave the real and imaginary parts of the complex symbols at the transmitter such that they go through independently fading channels. For CI to be effective, no two signal points in the signal set $\mathcal{X}$ should have the same co-ordinate. This condition can be met by rotating the standard $M$-QAM signal sets [15], [16]. In the following, we assume that the signal sets $\left\{\mathcal{X}_{k}\right\}_{k=1}^{K}$ are appropriately rotated. The effect of rotation angle and the optimal angle of rotation are discussed in subsequent sections.

\section{A. CISM Transceiver}

- Interleave real and imaginary parts of $\left\{x_{k}\right\}_{k=1}^{K}(K \leq n)$ to obtain $\left\{\tilde{x}_{k}\right\}_{k=1}^{K}$, where,

$$
\tilde{x}_{k}=\Re x_{k}+\jmath \Im x_{K-(k-1)}
$$

- Transmit $\mathbf{s}=\mathbf{V}_{K} \mathbf{P} \tilde{\mathbf{x}}$, where $\tilde{\mathbf{x}}=\left[\begin{array}{lll}\tilde{x}_{1} & \tilde{x}_{2} \ldots \tilde{x}_{K}\end{array}\right]^{T}$

- Receive $\tilde{\mathbf{y}}=\mathbf{H s}+\mathbf{n}=\mathbf{H V}_{K} \mathbf{P} \tilde{\mathbf{x}}+\mathbf{n}$

- Obtain $\tilde{\mathbf{r}}=\mathbf{U}_{K}^{H} \tilde{\mathbf{y}}=\boldsymbol{\Lambda}_{K} \mathbf{P} \tilde{\mathbf{x}}+\mathbf{w}$, where $\boldsymbol{\Lambda}_{K}=$ $\operatorname{diag}\left(\left\{\sqrt{\lambda_{k}}\right\}_{k=1}^{K}\right)$ and $\mathbf{w}=\mathbf{U}_{K}^{H} \mathbf{n}$.

$$
\Rightarrow \tilde{r}_{k}=\sqrt{\lambda_{k} p_{k}} \tilde{x}_{k}+w_{k}, k=1, \ldots, K
$$

- De-interleave $\tilde{r}_{k}$ to obtain $r_{k}$ :

$$
\begin{gathered}
r_{k}=\Re \tilde{r}_{k}+\jmath \Im \tilde{r}_{K-(k-1)}, k=1, \ldots, K \\
\Rightarrow r_{k}=\sqrt{\lambda_{k} p_{k}} \Re x_{k}+\jmath \sqrt{\lambda_{K-(k-1)} p_{K-(k-1)}} \Im x_{k} \\
+\Re w_{k}+\jmath \Im w_{K-(k-1)}
\end{gathered}
$$

- Estimate $x_{k}, k=1, \ldots, K$ :

$$
\begin{aligned}
\hat{x}_{k}=\arg \min _{x_{k} \in \mathcal{X}_{k}} \mid & r_{k}-\left(\sqrt{\lambda_{k} p_{k}} \Re x_{k}\right. \\
& \left.+\jmath \sqrt{\lambda_{K-(k-1)} p_{K-(k-1)}} \Im x_{k}\right)\left.\right|^{2}
\end{aligned}
$$


As can be seen from (7), the received signals gets decoupled and hence can be decoded by single-symbol maximum likelihood (ML) decoding. It is easy to verify that the received signal power on the interleaved $k^{t h}$ and $(K-(k-1))^{t h}$ eigenmodes is the same and hence, $\mathcal{X}_{k}$ and $\mathcal{X}_{K-(k-1)}$ are assumed to be the same. Among the $K$ eigenmodes used for transmission, the two symbols transmitted on strongest and weakest eigenmodes are interleaved and the two symbols transmitted on second strongest and second weakest eigenmodes are interleaved and so on. When $K$ is an odd number, symbols transmitted on $\left(\frac{K+1}{2}\right)^{t h}$ eigenmode will not get affected by the interleaving.

\section{DIVERSITY GAIN OF CISM}

The error rate performance of CISM, in particular, the diversity gain, is analyzed in this section. We consider uncoded transmission over $K \leq n$ eigenmodes with ${ }^{1} p_{k}=P / K, k=$ $1, \ldots, K$, and assume $\mathcal{X}_{k}=\mathcal{X}_{K-(k-1)}$. MIMO channels with $\operatorname{rank}(\mathbf{H})=2$ are considered in the foolowing and $\operatorname{rank}(\mathbf{H})>2$ case is deferred to section $\mathrm{V}$.

A. MIMO Channels with $\operatorname{rank}(\mathbf{H})=2$

Consider $\mathbf{H} \in \mathbb{C}^{N_{r} \times N_{t}}$ with $n=\min \left\{N_{t}, N_{r}\right\}=2$. When $\left\{h_{i j}\right\}$ are i.i.d and $h_{i j} \sim \mathcal{C N}(0,1), \operatorname{rank}(\mathbf{H})=n=2$ with probability one. For illustrative purpose, we consider 4-QAM signaling and analysis for any $M$-QAM can be done in a similar way.

Let $\mathcal{X}_{1}=\mathcal{X}_{2}=\mathcal{X}=\left\{x_{A}, x_{B}, x_{C}, x_{D}\right\}$ where $x_{A}=$ $e^{j \theta}\left(\frac{1}{2}+\jmath \frac{1}{2}\right), x_{B}=e^{j \theta}\left(-\frac{1}{2}+\jmath \frac{1}{2}\right), x_{C}=-x_{A}$ and $x_{D}=-x_{B}$. Let $P_{s, k}^{4-\text { QAM }}$ denote the SEP on $k^{\text {th }}$ eigenmode and $P_{s}^{4 \text {-QAM }}$ be the average SEP, averaged over both the eigenmodes.

Result 1: $P_{s, 1}^{4-\mathrm{QAM}}=P_{s, 2}^{4-\mathrm{QAM}}=P_{s}^{4-\mathrm{QAM}}$ and

$P_{s}^{4-\mathrm{QAM}} \leq \operatorname{Pr}\left\{x_{A} \rightarrow x_{B}\right\}+\operatorname{Pr}\left\{x_{A} \rightarrow x_{C}\right\}+\operatorname{Pr}\left\{x_{A} \rightarrow x_{D}\right\}$

where

$$
\begin{aligned}
& \operatorname{Pr}\left\{x_{A} \rightarrow x_{B}\right\}=\operatorname{Pr}\left\{x_{A} \rightarrow x_{D}\right\}= \\
& \frac{m}{2 \pi} \int_{0}^{\frac{\pi}{2}} \frac{1}{\left(1+\frac{\mathrm{SNR}}{8 \sin ^{2} t} \cos ^{2} \theta\right)^{m+1}\left(1+\frac{\mathrm{SNR}}{8 \sin ^{2} t} \sin ^{2} \theta\right)^{m-1}} d t \\
& +\frac{m}{2 \pi} \int_{0}^{\frac{\pi}{2}} \frac{1}{\left(1+\frac{\mathrm{SNR}}{8 \sin ^{2} t} \cos ^{2} \theta\right)^{m-1}\left(1+\frac{\mathrm{SNR}}{8 \sin ^{2} t} \sin ^{2} \theta\right)^{m+1}} d t \\
& -\frac{m-1}{\pi} \int_{0}^{\frac{\pi}{2}} \frac{1}{\left(1+\frac{\mathrm{SNR}}{8 \sin ^{2} t} \cos ^{2} \theta\right)^{m}\left(1+\frac{\mathrm{SNR}}{8 \sin ^{2} t} \sin ^{2} \theta\right)^{m}} d t
\end{aligned}
$$

and

$$
\begin{aligned}
& \operatorname{Pr}\left\{x_{A} \rightarrow x_{C}\right\}= \\
& \quad \frac{m}{2 \pi} \int_{0}^{\frac{\pi}{2}} \frac{1}{\left(1+\frac{\mathrm{SNR}}{8 \sin ^{2} t} \psi_{1}\right)^{m+1}\left(1+\frac{\mathrm{SNR}}{8 \sin ^{2} t} \psi_{2}\right)^{m-1}} d t \\
& +\frac{m}{2 \pi} \int_{0}^{\frac{\pi}{2}} \frac{1}{\left(1+\frac{\mathrm{SNR}}{8 \sin ^{2} t} \psi_{1}\right)^{m-1}\left(1+\frac{\mathrm{SNR}}{8 \sin ^{2} t} \psi_{2}\right)^{m+1}} d t \\
& -\frac{m-1}{\pi} \int_{0}^{\frac{\pi}{2}} \frac{1}{\left(1+\frac{\mathrm{SNR}}{8 \sin ^{2} t} \psi_{1}\right)^{m}\left(1+\frac{\mathrm{SNR}}{8 \sin ^{2} t} \psi_{2}\right)^{m}} d t
\end{aligned}
$$

${ }^{1}$ As power allocation effects only the coding gain but not the diversity gain [10], we consider uniform power allocation. where $\psi_{1}=1-\sin 2 \theta$ and $\psi_{2}=1+\sin 2 \theta$.

Proof: See Appendix A.

At high SNR, assuming $\sin 2 \theta \neq 0$ and $|\sin 2 \theta| \neq 1$, the pairwise error probabilities (PEPs) given above can be approximated as follows.

$$
\operatorname{Pr}\left\{x_{A} \rightarrow x_{B}\right\} \approx \kappa C_{A B} \mathrm{SNR}^{-2 m}
$$

where $\kappa=\frac{8^{2 m} m}{4}\left(\frac{4 m-1}{4 m} \frac{4 m-3}{4 m-2} \cdots \frac{1}{2}\right)$ and

$$
\begin{array}{r}
C_{A B}=\frac{1}{\left(\cos ^{2} \theta\right)^{m+1}\left(\sin ^{2} \theta\right)^{m-1}}+\frac{1}{\left(\cos ^{2} \theta\right)^{m-1}\left(\sin ^{2} \theta\right)^{m+1}} \\
-\frac{2(m-1)}{m} \frac{1}{\left(\cos ^{2} \theta \sin ^{2} \theta\right)^{m}}
\end{array}
$$

Similarly,

$$
\operatorname{Pr}\left\{x_{A} \rightarrow x_{C}\right\} \approx \kappa C_{A D} \mathrm{SNR}^{-2 m}
$$

where

$$
C_{A D}=\frac{1}{\psi_{1}^{m+1} \psi_{2}^{m-1}}+\frac{1}{\psi_{1}^{m-1} \psi_{2}^{m+1}}-\frac{2(m-1)}{m} \frac{1}{\left(\psi_{1} \psi_{2}\right)^{m}}
$$

Hence, at high SNRs,

$$
P_{s}^{4-\mathrm{QAM}} \leq \kappa\left(2 C_{A B}+C_{A D}\right) \mathrm{SNR}^{-2 m}
$$

$\theta_{\text {opt }}$, the optimal rotation angle, is computed by maximizing the coding gain $\left(2 C_{A B}+C_{A D}\right)$. For example, when $m=2$, $\theta_{\text {opt }}=27.9^{\circ}$ and for $m=4, \theta_{\text {opt }}=29.15^{\circ}$.

$P_{s, 1}$ is lower bounded by the PEP corresponding to confusing $x_{A}$ with its nearest neighbor.

$$
\operatorname{Pr}\left\{x_{A} \rightarrow x_{B}\right\}<P_{s, 1}^{4-\mathrm{QAM}} \leq\left(\text { Union bound on } P_{s, 1}^{4-\mathrm{QAM}}\right)
$$

From (12) and (14), we see that,

$\kappa C_{A B} \mathrm{SNR}^{-2 m}<P_{s, 1}^{4-\mathrm{QAM}} \leq \kappa\left(C_{A B}+C_{A C}+C_{A D}\right) \mathrm{SNR}^{-2 m}$

As both the upper bound and lower bound on $P_{s, 1}^{4-\mathrm{QAM}}$ have the same SNR exponent, it follows that data transmitted on first eigenmode has a diversity gain of $2 \mathrm{~m}$. By a similar argument, we can show that $P_{s, 2}^{4-\mathrm{QAM}}$ decays as $\mathrm{SNR}^{-2 m}$ and hence the overall diversity gain is $2 m$. SEP analysis for any $M$ QAM can be carried out in a similar way to show that CISM always achieves the maximum diversity gain $2 m$ offered by the channel when $\operatorname{rank}(\mathbf{H})=2$.

When the input symbols are from rotated BPSK, i.e., when $\mathcal{X}_{1}=\mathcal{X}_{2}=\mathcal{X}=\left\{e^{\jmath \theta},-e^{\jmath \theta}\right\}$, we compute exact SEP without resorting to union bound and is given below.

$$
\begin{aligned}
& P_{s, 1}^{\mathrm{BPSK}}=P_{s, 2}^{\mathrm{BPSK}}=P_{s}^{\mathrm{BPSK}}= \\
& \frac{m}{2 \pi} \int_{0}^{\frac{\pi}{2}} \frac{1}{\left(1+\frac{\mathrm{SNR}}{4 \sin ^{2} t} \cos ^{2} \theta\right)^{m+1}\left(1+\frac{\mathrm{SNR}}{4 \sin ^{2} t} \sin ^{2} \theta\right)^{m-1}} d t \\
+ & \frac{m}{2 ! \pi} \int_{0}^{\frac{\pi}{2}} \frac{1}{\left(1+\frac{\mathrm{SNR}}{4 \sin ^{2} t} \cos ^{2} \theta\right)^{m-1}\left(1+\frac{\mathrm{SNR}}{4 \sin ^{2} t} \sin ^{2} \theta\right)^{m+1}} d t \\
- & \frac{m-1}{\pi} \int_{0}^{\frac{\pi}{2}} \frac{1}{\left(1+\frac{\mathrm{SNR}}{4 \sin ^{2} t} \cos ^{2} \theta\right)^{m}\left(1+\frac{\mathrm{SNR}}{4 \sin ^{2} t} \sin ^{2} \theta\right)^{m}} d t
\end{aligned}
$$

Numerical evaluation of $\theta_{\text {opt }}$ results in $\theta_{\text {opt }}=45^{\circ}, \forall m \in \mathbb{N}$. 


\section{Diversity-Multiplexing Tradeoff of CiSM}

In the following, we compute the SEP $P_{s, k}$ when $R_{k}$, data rate on $k^{\text {th }}$ eigenmode, scales with SNR as $R_{k}=r_{k} \log$ SNR $\mathrm{b} / \mathrm{s} / \mathrm{Hz}, r_{k} \in[0,1]$, and obtain the DMT of CISM.

Result 2: The symbol error probability of $k^{t h}$ eigenmode at a multiplexing gain of $r$ is given by

$$
P_{s, k} \doteq \mathrm{SNR}^{-g_{d}^{\operatorname{cISM}}(k)\left(1-r_{k}\right)} \quad r_{k} \in[0,1]
$$

where

$$
g_{d}^{\mathrm{CISM}}(k)=\max \left\{g_{d}(k), g_{d}(K-(k-1))\right\}
$$

and $g_{d}(k)=(m-k+1)(n-k+1)$.

Proof: See Appendix B.

The classical diversity gain of $k^{t h}$ data stream can be obtained by making $r_{k}=0$ in (18).

Remark 1: The (classical) diversity gain of $k^{\text {th }}$ data stream in CISM is given by

$$
g_{d}^{\text {cISM }}(k)=(m-i+1)(n-i+1), k=1, \ldots, K
$$

where $i=\min \{k, K-(k-1)\}$. It follows from (3) and (19). Note that $g_{d}^{\text {CISM }}(k)=g_{d}^{\text {CISM }}(K-(k-1))$. Letting $n=2$ and $K=2$, it can be noticed that remark 1 is consistent with the results obtained in section IV. Remark 1 shows that, coordinate interleaving two data streams transmitted over two eigenmodes having different diversity gains would make the diversity gain of both the streams equal to the diversity gain of stronger eigenmode. Thus, CISM improves the diversity gains of the weaker eigenmodes resulting in a significant gain in the overall diversity, as shown by the following remark.

Remark 2: The overall (classical) diversity gain of CISM with $K \leq n$ is given by

$$
g_{d}^{\mathrm{cISM}}=\left(m-\left\lfloor\frac{K+1}{2}\right\rfloor+1\right)\left(n-\left\lfloor\frac{K+1}{2}\right\rfloor+1\right)
$$

SVDTR, as well as many advanced diagonal transceivers such as those proposed in [8], have an overall diversity gain of $g_{d}=$ $(m-K+1)(n-K+1)[10]$. Hence, for any choice of $K, 1<$ $K \leq n$, CISM results in a significantly higher diversity gain than existing diagonal transceivers. When $K=1$, both CISM and SVDTR reduces to MEBF. Now, we proceed to determine the DMT achieved by CISM.

When $K=n=2, P_{s, 1} \doteq \mathrm{SNR}^{-2 m\left(1-r_{1}\right)}$ and $P_{s, 2} \doteq$ $\mathrm{SNR}^{-2 m\left(1-r_{2}\right)}$. Dividing the total input data rate $R=$ $r \log$ SNR, $r \in[0,2]$, equally between the two eigenmodes results in the following.

Remark 3: Over rank 2 MIMO channels, CISM achieves the DMT given by,

$$
d^{\text {cISM }}(r)=2 m(1-r / 2), \quad r \in[0,2]
$$

Fig. 1 shows the DMT for $4 \times 2$ MIMO channel. The tradeoff for SVDTR (or, multiple beamforming), and MEBF are derived in [12]. The figure also shows the optimal tradeoff of the MIMO channel with coding across space and time ("Optimal tradeoff") and "space-only coding" without CSI at the transmitter, derived in [2]. The UCD-VBLAST transceiver achieves the optimal open-loop tradeoff [9] and hence, the "Optimal tradeoff" curve also shows the DMT of UCDVBLAST. Note that CISM is space-only coding with CSI at the transmitter.

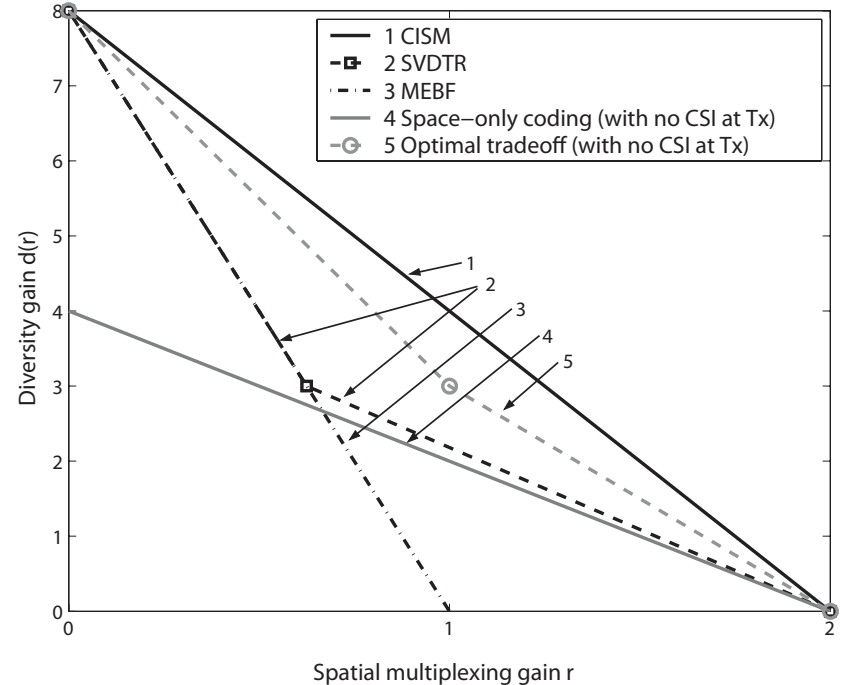

Fig. 1. Diversity-Multiplexing tradeoff comparisons of different schemes over $4 \times 2$ MIMO channel.

When $n>2$ and $K>2$, CISM results in un-equal diversity gains $\left\{g_{d}^{\operatorname{cISM}}(k)\right\}_{k=1}^{K}$ and the overall diversity gain is determined by $\min \left\{g_{d}^{\operatorname{Clsm}}(k)\right\}_{k=1}^{K}$. With uniform rate allocation $\left(r_{k}=r / K, k=1, \ldots, K\right)$ across all the $K$ active eigenmodes,

$$
d^{\operatorname{cISM}}(r)=g_{d}^{\operatorname{cISM}}(i)(1-r / K), \quad r \in[0, K]
$$

where $i=\left\lfloor\frac{K+1}{2}\right\rfloor$ and $g_{d}^{\text {clsM }}(i)$ is given by (20). The tradeoff can be improved by finding the optimal number of active eigenmodes $\left(K^{*}\right)$ for each input rate $r$, and by optimally distributing the rate across the active modes. We note that, a similar analysis has been reported in [12] for multiple beamforming. Define $G_{d}^{\mathrm{CISM}}(2 k-1):=g_{d}^{\mathrm{CISM}}(k)$ for $k=$ $1, \ldots,\left\lfloor\frac{n+1}{2}\right\rfloor$ and $G_{d}^{\operatorname{cism}}(2 k):=g_{d}^{\operatorname{cism}}(k)$ for $k=1, \ldots,\left\lfloor\frac{n}{2}\right\rfloor$ and $G_{d}^{\operatorname{cISM}}(n+1):=0$. Let $D(K, r)=G_{d}^{\text {CISM }}(1)\left(1-r_{1}\right)=$ $\cdots=G_{d}^{\operatorname{cism}}(K)\left(1-r_{K}\right), K \leq n$. We need to find $K^{*}=$ $\max _{K} D(K, r)$ subject to the constraint $\sum_{k=1}^{K^{*}} r_{k}=r$. This results in

$$
K^{*}=\arg \max _{K} D(K, r)=\arg \max _{K}\left(\frac{K-r}{\sum_{i=1}^{K}\left(1 / G_{d}^{\mathrm{CISM}}(i)\right)}\right)
$$

with optimal rate allocation given by

$$
r_{k}=1-\frac{D\left(K^{*}, r\right)}{G_{d}^{\text {cISM }}(k)}, \quad k=1, \ldots, K^{*}
$$

Connecting the points $(r(k), d(k))$, where

$r(k)= \begin{cases}0 & k=0 \\ k-G_{d}^{\mathrm{CISM}}(k+1)\left(\sum_{i=1}^{k} 1 / G_{d}^{\mathrm{CISM}}(i)\right) & 0<k<n \\ n & k=n\end{cases}$

and

$$
\mathrm{d}(k)=G_{d}^{\text {CISM }}(k+1) \quad k=0, \ldots, n
$$

gives the DMT curve. Transition points are obtained by equating $D(i, \mathrm{r}(i))=D(i+1, \mathrm{r}(i)), i=1, \ldots, n-1$. 


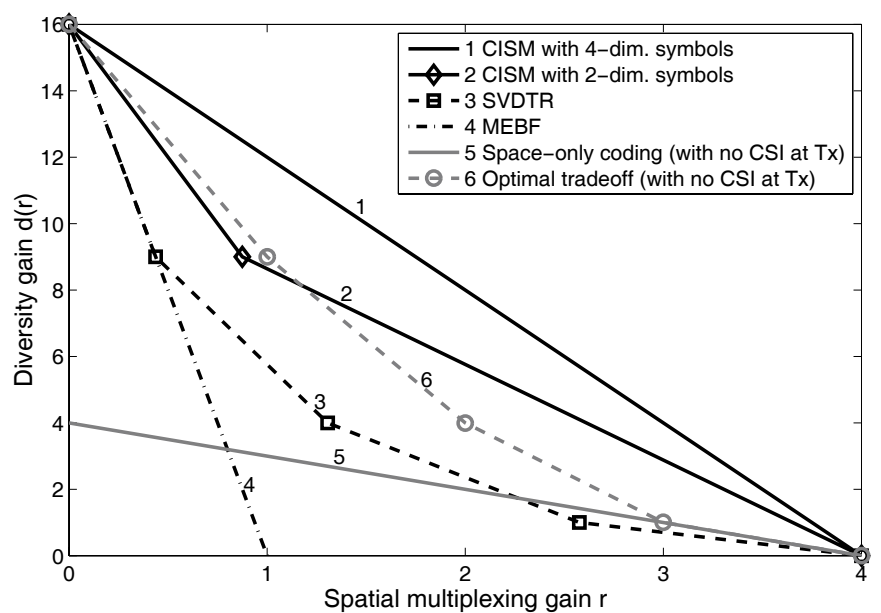

Fig. 2. Diversity-Multiplexing tradeoff comparisons of different schemes over $4 \times 4$ MIMO channel.

\section{A. CISM with multi-dimensional signaling}

Intrigued by (22), we investigate the DMT of CISM over rank $n$ MIMO channels when the input symbols are from rotated $n$-dimensional QAM constellations. An $n$-dimensional QAM signal set is obtained as the Cartesian product of $n / 2$ two-dimensional QAM signal sets [16]. To send $b$ bits in $n$-dimensions, the $n$-dimensional constellation will have (at least) $2^{b}$ points. A symbol vector from a rotated $n$-dimensional constellation is denoted by $\underline{x}_{k}=\underline{v}_{k} \Phi, \underline{x}_{k} \in \mathbb{R}^{n}$, where, $\underline{v}_{k}=\left(v_{k}(1), \ldots, v_{k}(n)\right) \in \mathbb{Z}^{n}$ is the un-rotated $n$-dimensional QAM symbol and $\boldsymbol{\Phi}$ is a rotation matrix chosen such that $x_{k}(i) \neq x_{l}(i), 1 \leq k, l \leq 2^{b}, k \neq l$.

To achieve an overall input data rate of $R=r \log$ SNR $\mathrm{b} / \mathrm{s} / \mathrm{Hz}, r \in[0, n]$, we choose two $n$-dimensional symbols (say, $\underline{x}_{1}$ and $\underline{x}_{2}$ ) carrying $R / 2$ bits each. The symbols are interleaved to obtain $\tilde{\mathbf{x}} \in \mathbb{C}^{n}$ where

$$
\tilde{x}_{i}=x_{1}(i)+\jmath x_{2}(i), i=1, \ldots, n
$$

The received vector $\tilde{\mathbf{r}}=\Lambda \mathbf{P} \tilde{\mathbf{x}}+\mathbf{w}$ is de-interleaved to obtain $\underline{r}_{1}=\Re \tilde{\mathbf{r}}$ and $\underline{r}_{2}=\Im \tilde{\mathbf{r}}$ where

$$
r_{i}(j)=\sqrt{\lambda_{j} p_{j}} x_{i}(j)+w_{j}, i=1,2 ; j=1, \ldots, n
$$

$\underline{\hat{x}}_{i}$ are obtained from $\underline{r}_{i}$ through single ( $n$-dimensional) symbol ML decoding.

Result 3: DMT achieved by CISM over rank $n$ MIMO channels with input symbols from rotated $n$-dimensional constellations is given by

$$
d(r)=m n\left(1-\frac{r}{n}\right), \quad r \in[0, n]
$$

Proof: See Appendix C.

Fig. 2 compares DMT achieved by different schemes over $4 \times 4$ MIMO channel. DMT of CISM with 2 -dimensional signaling is plotted according to (26) and (27) and it outperforms SVDTR and MEBF. As shown by (30), DMT achieved by CISM with 4-dimensional input symbols is a straight line connecting the endpoints $(0,16)$ and $(4,0)$. Note that the improved tradeoff with $n$-dimensional symbols is achieved at the cost of higher decoding complexity.

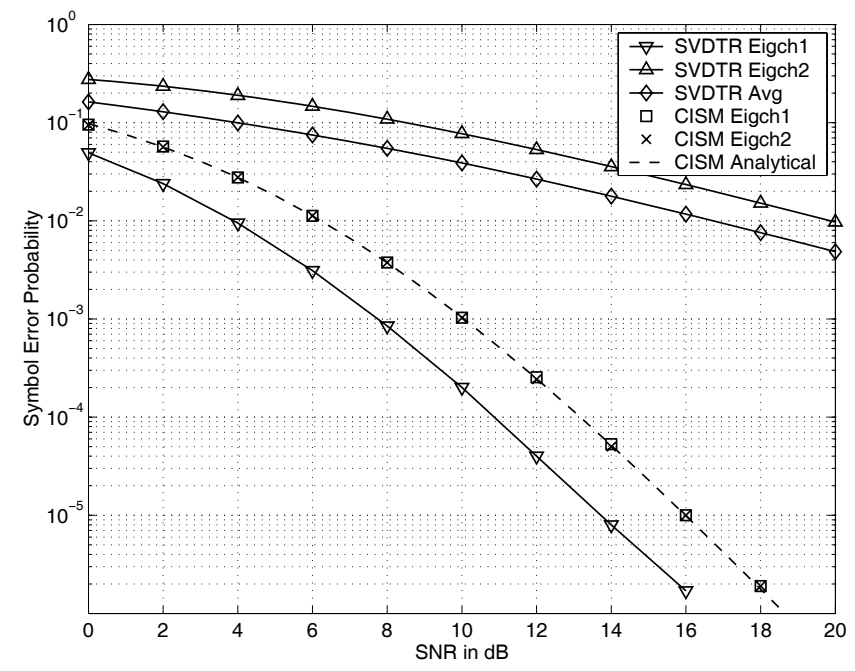

Fig. 3. Example 1: Symbol error probability of SVDTR and CISM over $2 \times 2$ MIMO channel with BPSK signaling. Note that CISM Avg = CISM Eigch1 = CISM Eigch2.

Result 3 may be interpreted as, in MIMO channels, it is possible to achieve a linear DMT connecting the end points $(0, m n)$ and $(n, 0)$ with space-only coding with perfect CSI at both ends. CISM achieves this with appropriate signaling.

\section{Simulation Results}

This section reports SEP of the proposed CISM transceiver, evaluated through Monte Carlo simulations. We consider block-fading channel and uncoded data transmission over $K=n$ eigenmodes with $p_{k}=P / n, k=1, \ldots, n$ and $P=1$.

Example 1: For $N_{t}=2$ and $N_{r}=2$, the SEP of SVDTR and CISM is plotted in Fig. 3. Data symbols are drawn from BPSK constellation rotated by $\theta_{\text {opt }}=45^{0}$. "SVDTR Eigch $k$ " and "CISM Eigch $k$ " refers to $k^{t h}$ eigen subchannel of SVDTR and CISM, respectively. "SVDTR Avg" and "CISM Avg" denote the overall SEP of SVDTR and CISM, respectively. In SVDTR, as shown in [10], the second eigenmode drastically degrades the overall SEP. In CISM, both the eigenmodes have equal SEP with $4^{\text {th }}$ order diversity. Analytical SEP, plotted by evaluating (17) with $m=2$, matches exactly with the simulation results.

Example 2: Consider CISM over $2 \times 2$ MIMO channel with data symbols from 4-QAM constellation rotated by $\theta_{\text {opt }}=27.9^{\circ}$. Note that $\theta_{\text {opt }}$ is different from $31.7175^{\circ}$ which is the optimal rotation angle when both the channels are Rayleigh fading channels with unit diversity gain [17]. Fig. 4 compares the SEP obtained through simulations with the union bound given by (9). It also shows the SEP of UCD-VBLAST which involves unitary precoding along with optimal power allocation [9]. Both UCD-VBLAST and CISM achieve maximum diversity gain of 4 but CISM (with uniform power allocation) has slightly lower coding gain. "CISM+PL" denotes CISM with power allocation given by $p_{k}=\frac{2 P}{K} \frac{\lambda_{k}}{\lambda_{k}+\lambda_{K-(k-1)}}, k=$ $1, \ldots, K$. It can be verified that this power allocation results in higher received SNR compared to uniform power allocation. The power allocation given above is a heuristic one and determining the optimal power allocation will be considered in the future work. Further, since the DMT of UCD-VBLAST 


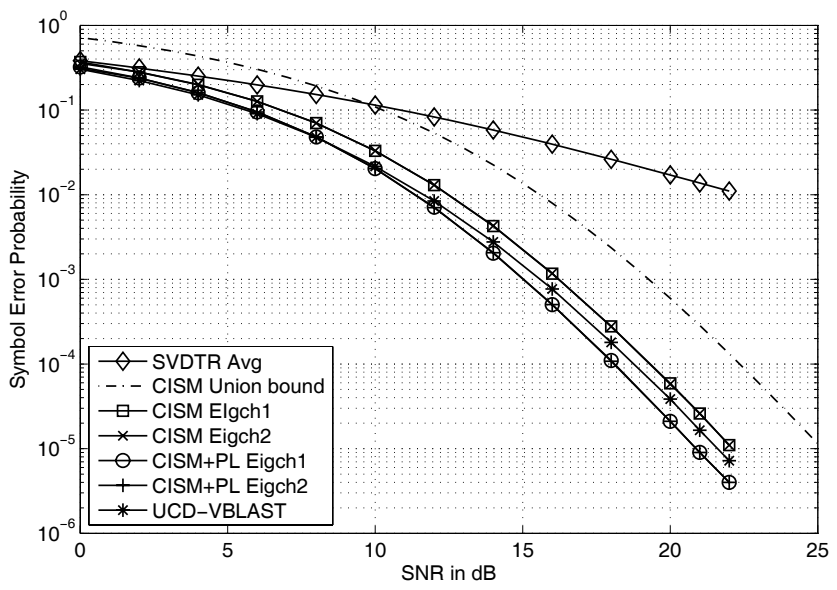

Fig. 4. Example 2: Symbol error probability of SVDTR and CISM over $2 \times 2$ MIMO channel with 4-QAM signaling. Note that CISM Avg=CISM Eigch1 =CISM Eigch2.

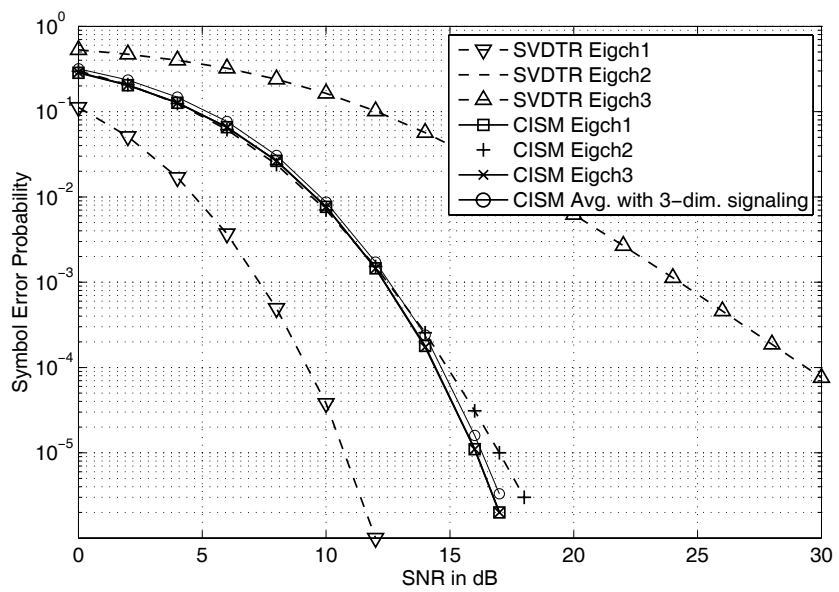

Fig. 5. Example 3: Symbol error probability of SVDTR and CISM over $4 \times 3$ MIMO channel with 4 -QAM signaling. Note that SVDTR Eigch2=CISM Eigch2 and CISM Eigch1=CISM Eigch3.

is same as that of optimal tradeoff without CSI at the transmitter, CISM achieves a higher $d(r)$ than UCD-VBLAST for $0<r<n$ (cf. Fig. 1).

Example 3: Fig. 5 shows SEP of CISM over $4 \times 3$ MIMO channel with input symbols from 4-QAM constellation rotated by $27^{\circ}$. Co-ordinates of the symbols transmitted on first and third eigenmodes are interleaved and hence, they have the same SEP. CISM Eigch1 and CISM Eigch3 are parallel to SVDTR Eigch1 showing that $g_{d}^{\text {CISM }}(1)=g_{d}^{\text {CISM }}(3)=g_{d}(1)=$ 12. This justifies remark 1 . As the data stream transmitted on second eigenmode does not get effected by interleaving, it has same SEP as SVDTR Eigch2. Hence, CISM with 2dimensional signaling achieves an overall diversity of $g_{d}^{\text {cisM }}=$ $g_{d}(2)=6$. "CISM Avg. with 3-dim. signaling" shows the average SEP of CISM when input symbols are from rotated 3-dimensional QAM constellation. As can be seen, SEP of 3dimensional signaling has the same slope as that of SVDTR Eigch1 and achieves the maximum diversity gain of 12 .

\section{CONCLUSIONS}

With perfect CSI at both ends of the link, a MIMO channel can be diagonalized and multiple data streams can be sent in parallel on the resulting eigenmodes. In most of the linear diagonal transceivers proposed to date, the weaker eigenmodes having low diversity gains drastically degrade the overall error rate performance. This paper proposed a novel transceiver, referred to as co-ordinate interleaved spatial multiplexing (CISM), that improves the diversity gains of the weaker eigenmodes. CISM diagonalizes the channel through SVD and interleaves the co-ordinates of the input symbols (from rotated QAM constellations) transmitted on strong and weak eigenmodes.

By computing the upper bound and lower bound on symbol error probability of the eigen sub-channels, the diversity gains of CISM have been determined. The analytical results, supported by simulation results, show that CISM achieves significantly higher overall diversity gain by improving the diversity of weaker eigenmodes. The diversity-multiplexing tradeoff analysis shows that, CISM achieves higher diversity gain at all multiplexing gains compared to the existing diagonal transceivers.

\section{APPENDIX A \\ SEP OF 4-QAM OVER RAYLEIGH MIMO CHANNELS $(\operatorname{RANK}(\mathbf{H})=2)$}

The joint pdf of the unordered eigenvalues of $\mathbf{W}$ is [18]

$$
\begin{aligned}
f\left(\lambda_{1}, \lambda_{2}\right)= & \left(2 ! K_{m, 2}\right)^{-1} e^{-\left(\lambda_{1}+\lambda_{2}\right)} \\
& \left(\lambda_{1}^{m} \lambda_{2}^{m-2}+\lambda_{1}^{m-2} \lambda_{2}^{m}-2 \lambda_{1}^{m-1} \lambda_{2}^{m-1}\right)
\end{aligned}
$$

where $K_{m, 2}=(m-1) !(m-2)$ ! is the normalizing constant. Union bound on $P_{s, 1}^{4-\mathrm{QAM}}$ is given by

$$
\begin{gathered}
P_{s, 1}^{4-\mathrm{QAM}} \leq \operatorname{Pr}\left\{x_{A} \rightarrow x_{B}\right\}+\operatorname{Pr}\left\{x_{A} \rightarrow x_{C}\right\}+\operatorname{Pr}\left\{x_{A} \rightarrow x_{D}\right\} \\
\operatorname{Pr}\left\{x_{A} \rightarrow x_{B} \mid \lambda_{1}, \lambda_{2}\right\}=Q\left(\frac{\left|u_{A}-u_{B}\right|}{2 \sigma}\right)
\end{gathered}
$$

where $u_{A}=\sqrt{\lambda_{1} p_{1}} \Re x_{A}+\jmath \sqrt{\lambda_{2} p_{2}} \Im x_{A}, u_{B}=\sqrt{\lambda_{1} p_{1}} \Re x_{B}+$ $\jmath \sqrt{\lambda_{2} p_{2}} \Im x_{B}$ and $Q(\cdot)$ is the Gaussian $Q$-function. Substituting $p_{1}=p_{2}=P / 2$ (uniform power allocation),

$\operatorname{Pr}\left\{x_{A} \rightarrow x_{B}\right\}=E_{\lambda_{1}, \lambda_{2}}\left[Q\left(\sqrt{\frac{P\left(\lambda_{1} \cos ^{2} \theta+\lambda_{2} \sin ^{2} \theta\right)}{4 \sigma^{2}}}\right)\right]$

Using alternative form of Gaussian $Q$-function [19] given by $Q(x)=\frac{1}{\pi} \int_{0}^{\frac{\pi}{2}} e^{\frac{-x^{2}}{2 \sin ^{2} t}} d t$, along with (31) and by recalling that $P / \sigma^{2}=$ SNR, (34) can be evaluated to obtain (10). Due to symmetry, $\operatorname{Pr}\left\{x_{A} \rightarrow x_{D}\right\}=\operatorname{Pr}\left\{x_{A} \rightarrow x_{B}\right\}$.

$$
\operatorname{Pr}\left\{x_{A} \rightarrow x_{C}\right\}=E_{\lambda_{1}, \lambda_{2}}\left[Q\left(\sqrt{\frac{P\left(\lambda_{1} \psi_{1}+\lambda_{2} \psi_{2}\right)}{4 \sigma^{2}}}\right)\right]
$$

where $\psi_{1}=1-\sin 2 \theta$ and $\psi_{2}=1+\sin 2 \theta \cdot \operatorname{Pr}\left\{x_{A} \rightarrow x_{C}\right\}$ can be evaluated, similar to $\operatorname{Pr}\left\{x_{A} \rightarrow x_{B}\right\}$, to get (11). Union bound on $P_{s, 1}^{4-\mathrm{QAM}}$ can be computed by substituting (10) and (11) in (32). In a similar way, the union bound on $P_{s, 2}^{4-\mathrm{QAM}}$ can be computed to show that it is equal to the union bound on $P_{s, 1}^{4-\mathrm{QAM}}$. This proves result 1 . 


\section{APPENDIX B}

SEP OF $k^{t h}$ EIGENMODE WHEN $R_{k}=r_{k} \log$ SNR B/S/Hz

Let the input symbols are from a rotated QAM signal set whose size increases with SNR to satisfy $R_{k}=r_{k} \log$ SNR $\mathrm{b} / \mathrm{s} / \mathrm{Hz}$. Assuming symbol $x_{l}$ is transmitted, at high SNRs,

$$
\operatorname{Pr}\left\{x_{l} \rightarrow x_{\eta(l)}\right\}<\operatorname{Pr}\left\{\text { error } \mid x_{l} \text { sent }\right\} \leq \sum_{x_{i} \in \mathrm{X}_{l}} \operatorname{Pr}\left\{x_{l} \rightarrow x_{i}\right\}
$$

where $x_{\eta(l)}$ is a nearest neighbor to $x_{l}$ and $\mathrm{X}_{l} \subset e^{\jmath \theta} \mathcal{X}$ is the set of nearest neighbors to $x_{l}$.

$$
\operatorname{Pr}\left\{x_{l} \rightarrow x_{i} \mid \lambda_{k}, \lambda_{K-(k-1)}\right\}=Q\left(\frac{\left|u_{l}-u_{i}\right|}{2 \sigma}\right)
$$

where $u_{l}=\sqrt{\lambda_{k} p_{k}} \Re x_{l}+\jmath \sqrt{\lambda_{K-(k-1)} p_{K-(k-1)}} \Im x_{l}$, and $u_{i}=\sqrt{\lambda_{k} p_{k}} \Re x_{i}+\jmath \sqrt{\lambda_{K-(k-1)} p_{K-(k-1)}} \Im x_{i}$. To evaluate $\operatorname{Pr}\left\{x_{l} \rightarrow x_{i}\right\}$, we need $f\left(\lambda_{k}, \lambda_{K-(k-1)}\right)$, the joint pdf of $k^{\text {th }}$ and $(K-(k-1))^{t h}$ ordered eigenvalues of $\mathbf{W}$. For a central Wishart matrix $\mathbf{W}$, only the joint pdf of all its eigenvalues is known $^{2}$ [18]. Hence, we make use of two results from [10] and [13] to evaluate $\operatorname{Pr}\left\{x_{l} \rightarrow x_{i}\right\}$. As the symbols are from a rotated QAM constellation $e^{\jmath \theta} \mathcal{X}_{k}$,

$$
\begin{aligned}
& \Re x_{l}=a_{l} \cos \theta-b_{l} \sin \theta \\
& \Im x_{l}=a_{l} \sin \theta+b_{l} \cos \theta
\end{aligned}
$$

where $a_{l}+\jmath b_{l} \in \mathcal{X}_{k}$ (un-rotated QAM constellation) and $\theta$ is chosen such that $\Re x_{l} \neq \Re x_{i}$ and $\Im x_{l} \neq \Im x_{i}, \forall x_{l}, x_{i} \in$ $e^{\jmath \theta} \mathcal{X}_{k}, l \neq i$. With uniform power allocation and using (38),

$$
\begin{gathered}
\left|u_{l}-u_{i}\right|=\left[\frac { P } { K } \left(\lambda_{k}\left(\cos \theta\left(a_{l}-a_{i}\right)-\sin \theta\left(b_{l}-b_{i}\right)\right)^{2}\right.\right. \\
\left.\left.\quad+\lambda_{K-(k-1)}\left(\cos \theta\left(b_{l}-b_{i}\right)+\sin \theta\left(a_{l}-a_{i}\right)\right)^{2}\right)\right]^{\frac{1}{2}}
\end{gathered}
$$

Consider $\left(a_{l}-a_{i}\right)$ and $\left(b_{l}-b_{i}\right)$. While transmitting at $R_{k}$ $\mathrm{b} / \mathrm{s} / \mathrm{Hz}$, there are $2^{R_{k}}$ points in the QAM constellation and, in each of the real and imaginary dimensions, there are $2^{\frac{R_{k}}{2}}$ points. Distance between two adjacent points is $1 / 2^{\frac{R_{k}}{2}}$ (recall that $\mathcal{X}_{k}$ has unit energy). Assuming that $x_{l}$ and $x_{i}$ are $d_{r e}^{l i}$ and $d_{i m}^{l i}$ symbols apart along the real and imaginary dimension, respectively, $\left(a_{l}-a_{i}\right)$ is of the order of $d_{r e}^{l i} / 2^{\frac{R_{k}}{2}}$ and $\left(b_{l}-b_{i}\right)$ is of the order of $d_{i m}^{l i} / 2^{\frac{R_{k}}{2}}$ [14].

Without loss of generality, assume $k<K-(k-1)$, which implies $0<\lambda_{K-(k-1)} \leq \lambda_{k}$. We bound $\left|u_{l}-u_{i}\right|$ as, $\sqrt{\lambda_{k} 2^{-R_{k}} c_{1} P / K}<\left|u_{l}-u_{i}\right| \leq \sqrt{\lambda_{k} 2^{-R_{k}} c_{2} P / K}$ where $c_{1}=\cos ^{2} \theta\left(d_{r e}^{l i}\right)^{2}+\sin ^{2} \theta\left(d_{i m}^{l i}\right)^{2}-2 \cos \theta \sin \theta d_{r e}^{l i} d_{i m}^{l i}$ and $c_{2}=\left(d_{r e}^{l i}\right)^{2}+\left(d_{i m}^{l i}\right)^{2}$. Using the bounds on $\left|u_{l}-u_{i}\right|$ and noting that $R_{k}=r_{k} \log \mathrm{SNR}$ and $P / \sigma^{2}=\mathrm{SNR}$, we get

$$
\begin{array}{r}
E_{\lambda_{k}}\left[Q\left(\sqrt{\frac{\lambda_{k} \mathrm{SNR}^{\left(1-r_{k}\right)} c_{2}}{4 K}}\right)\right] \leq \operatorname{Pr}\left\{x_{l} \rightarrow x_{i}\right\}< \\
E_{\lambda_{k}}\left[Q\left(\sqrt{\frac{\lambda_{k} \mathrm{SNR}^{\left(1-r_{k}\right)} c_{1}}{4 K}}\right)\right]
\end{array}
$$

\footnotetext{
${ }^{2}$ When $n=2$ and $K=2, f\left(\lambda_{1}, \lambda_{2}\right)$ is the joint pdf of all eigenvalues of $\mathbf{W}$ and $P_{s, k}, k=1,2$, can be obtained directly by taking expectation over $f\left(\lambda_{1}, \lambda_{2}\right)$ as done in appendix A.
}

Bounds on $P_{s, k}$ depend only on $f\left(\lambda_{k}\right)$. First order expansion of $f\left(\lambda_{k}\right)$, derived in [10], is given by

$$
f\left(\lambda_{k}\right)=a_{k} \lambda_{k}^{d_{k}}+o\left(\lambda_{k}^{d_{k}}\right) \quad k=1, \ldots, n
$$

where

$$
d_{k}=(m-k+1)(n-k+1)-1
$$

(see Thm. 1 in [10] for $a_{k}$. It has no effect on diversity gain.)

Using (41) in (40) and using proposition 1 from [13], we get $P_{l i}^{\mathrm{LB}} \leq \operatorname{Pr}\left\{x_{l} \rightarrow x_{i}\right\} \leq P_{l i}^{\mathrm{UB}}$, where

$$
\begin{aligned}
& P_{l i}^{\mathrm{LB}}=C\left(\frac{c_{2}}{4 K} \mathrm{SNR}^{1-r_{k}}\right)^{-\left(d_{k}+1\right)}+o\left(\left(\mathrm{SNR}^{1-r_{k}}\right)^{-\left(d_{k}+1\right)}\right) \\
& P_{l i}^{\mathrm{UB}}=C\left(\frac{c_{1}}{4 K} \mathrm{SNR}^{1-r_{k}}\right)^{-\left(d_{k}+1\right)}+o\left(\left(\mathrm{SNR}^{1-r_{k}}\right)^{-\left(d_{k}+1\right)}\right)
\end{aligned}
$$

Since both the upper bound and lower bound on $\operatorname{Pr}\left\{x_{l} \rightarrow x_{i}\right\}$ have the same SNR exponent,

$$
\operatorname{Pr}\left\{x_{l} \rightarrow x_{i}\right\}=C_{l i}^{k} \mathrm{SNR}^{-\left(d_{k}+1\right)\left(1-r_{k}\right)}
$$

where $C_{l i}^{k}$ is the coding gain. It is easy to show that every PEP has the same SNR exponent. Thus, both the union bound and the lower bound on $\operatorname{Pr}\left\{\right.$ error $\mid x_{l}$ sent $\}$ in (36) decays as $\mathrm{SNR}^{-\left(d_{k}+1\right)\left(1-r_{k}\right)}$. Hence, $\operatorname{Pr}\left\{\right.$ error $\mid x_{l}$ sent $\}=$ $C_{l}^{k} \mathrm{SNR}^{-\left(d_{k}+1\right)\left(1-r_{k}\right)}$ and,

$$
\begin{aligned}
P_{s, k} & =\sum_{l=1}^{2^{R_{k}}} \operatorname{Pr}\left\{\text { error } \mid x_{l} \text { sent }\right\} \cdot \operatorname{Pr}\left\{x_{l} \text { sent }\right\} \\
& \doteq \mathrm{SNR}^{-\left(d_{k}+1\right)\left(1-r_{k}\right)}
\end{aligned}
$$

where $\doteq$ is the exponential equality [2]. Similarly,

$$
P_{s, K-(k-1)} \doteq \mathrm{SNR}^{-\left(d_{k}+1\right)\left(1-r_{K-(k-1)}\right)}
$$

when $R_{K-(k-1)}=r_{K-(k-1)} \log \mathrm{SNR} \mathrm{b} / \mathrm{s} / \mathrm{Hz}$. As $P_{s, k}=$ $P_{s, K-(k-1)}, R_{k}$ can be made equal to $R_{K-(k-1)}$. From (42) and (3), $d_{k}+1=g_{d}(k)$. Hence, result 2 is proved.

\section{APPENDiX C \\ PROOF OF RESULT 3}

Let $\underline{x}_{i}$ and $\underline{x}_{k}$ are the two $n$-dimensional symbols transmitted at a given time. First we find $\operatorname{Pr}\left\{\underline{\hat{x}}_{i} \neq \underline{x}_{i}\right\}$.

$$
\operatorname{Pr}\left\{\underline{x}_{i} \rightarrow \underline{x}_{j} \mid \underline{\lambda}=\left(\lambda_{1}, \ldots, \lambda_{n}\right)\right\}=Q\left(\frac{\left|\underline{u}_{i}-\underline{u}_{j}\right|}{2 \sigma}\right)
$$

where $\left|\underline{u}_{i}-\underline{u}_{j}\right|=\sqrt{\sum_{l=1}^{n} p_{l} \lambda_{l}\left(x_{i}(l)-x_{j}(l)\right)^{2}}$. Note that $x_{i}(l)=\sum_{t=1}^{n} \phi_{l t} v_{i}(t)$ and $x_{j}(l)=\sum_{t=1}^{n} \phi_{l t} v_{j}(t)$ and hence

$$
\left(x_{i}(l)-x_{j}(l)\right)^{2}=\sum_{t=1}^{n} \phi_{l t}^{2}\left(v_{i}(t)-v_{j}(t)\right)^{2}
$$

To meet a total input data rate of $R=r \log \mathrm{SNR} \mathrm{b} / \mathrm{s} / \mathrm{Hz} /$, $r \leq n$, the symbols $\underline{x}_{i}$ and $\underline{x}_{k}$ should carry $R / 2$ bits each, and hence, the $n$-dimensional constellation will have $2^{R / 2}$ points, with $2^{R / 2 n}$ points in each dimension. The distance between adjacent points is $2^{-R / 2 n}=\mathrm{SNR}^{-r / 2 n}$. Let $\underline{v}_{i}$ and $\underline{v}_{j}$ are $d_{t}^{i j}$ symbols apart along $t^{t h}$ dimension, so that $\left(v_{i}(t)-\right.$ $\left.v_{j}(t)\right)^{2}=\left(d_{t}^{i j}\right)^{2} \mathrm{SNR}^{-r / n}$. This implies, $\left(x_{i}(l)-x_{j}(l)\right)^{2}=$ $\sum_{t=1}^{n} \phi_{l t}^{2}\left(d_{t}^{i j}\right)^{2} \mathrm{SNR}^{-r / n}$. With $p_{l}=P / n, l=1 \ldots, n$,

$$
\operatorname{Pr}\left\{\underline{x}_{i} \rightarrow \underline{x}_{j}\right\}=E_{\underline{\lambda}}\left[Q\left(\sqrt{\frac{\sum_{l=1}^{n} \lambda_{l} \xi_{l} \mathrm{SNR}^{1-r / n}}{4 n}}\right)\right]
$$


where $\xi_{l}=\sum_{t=1}^{n} \phi_{l t}^{2}\left(d_{t}^{i j}\right)^{2}$ and $\xi_{l}>0$. As

$$
\lambda_{1} \xi_{1} \mathrm{SNR}^{1-r / n}<\sum_{l=1}^{n} \lambda_{l} \xi_{l} \mathrm{SNR}^{1-r / n} \leq \lambda_{1} \mathrm{SNR}^{1-r / n} \sum_{l=1}^{n} \xi_{l}
$$

$\operatorname{Pr}\left\{\underline{x}_{i} \rightarrow \underline{x}_{j}\right\}$ can be bounded as

$$
\begin{gathered}
E_{\lambda_{1}}\left[Q\left(\sqrt{\frac{\lambda_{1} \mathrm{SNR}^{1-r / n} \sum_{l=1}^{n} \xi_{l}}{4 n}}\right)\right] \leq \operatorname{Pr}\left\{\underline{x}_{i} \rightarrow \underline{x}_{j}\right\}< \\
E_{\lambda_{1}}\left[Q\left(\sqrt{\frac{\lambda_{1} \xi_{1} \mathrm{SNR}^{1-r / n}}{4 n}}\right)\right]
\end{gathered}
$$

(47) is similar to (40) and can be evaluated using (41), (42) and proposition 1 from [13] to show that $\operatorname{Pr}\left\{\underline{x}_{i} \rightarrow\right.$ $\left.\underline{x}_{j}\right\}=C_{i j}$ SNR $^{-\left(d_{1}+1\right)(1-r / n)}$. This results in $\operatorname{Pr}\left\{\underline{\hat{x}}_{i} \neq\right.$ $\left.\underline{x}_{i}\right\} \doteq \mathrm{SNR}^{-\left(d_{1}+1\right)(1-r / n)}$. Similarly, it can be shown that $\operatorname{Pr}\left\{\underline{\hat{x}}_{j} \neq \underline{x}_{j}\right\} \doteq \mathrm{SNR}^{-\left(d_{1}+1\right)(1-r / n)}$ and hence, overall SEP with $n$-dimensional signaling over $n$ eigenmodes is given by

$$
P_{s} \doteq \mathrm{SNR}^{-\left(d_{1}+1\right)(1-r / n)}, r \in[0, n]
$$

Noticing that $d_{1}+1=m n$ completes the proof.

\section{ACKNOWLEDGMENT}

The authors thank Gajanana Krishna for useful discussions and the anonymous reviewers whose suggestions improved the quality of presentation.

\section{REFERENCES}

[1] I. E. Telatar, "Capacity of multi-antenna Gaussian channels," Eur. Trans. Telecommun., vol. 10, no. 6, pp. 585-595, 1999.

[2] L. Zheng and D. N. C. Tse, "Diversity and multiplexing: a fundamental tradeoff in multiple-antenna channels," IEEE Trans. Inform. Theory, vol. 49, no. 5, pp. 1073-1096, May 2003.

[3] G. J. Foschini, "Layered space-time architecture for wireless communication in a fading environment when using multi-element antennas," AT\&T Bell Labs Tech. J., vol. 1, pp. 41-59, 1996.
[4] A. Scaglione, G. B. Giannakis, and J. M. Cioffi, "Redundant filterbank precoders and equalizers-part I: unification and optimal designs," IEEE Trans. Signal Processing, pp. 1988-2005, July 1999.

[5] H. Sampath, P. Stoica, and A. Paulraj, "Generalized linear precoder and decoder design for MIMO channels using the weighted MMSE criterion," IEEE Trans. Commun., vol. 49, pp. 2198-2206, Dec. 2001.

[6] A. Scaglione, P. Stoica, S. Barbarossa, and G. B. Giannakis, "Optimal designs for space-time linear precoders and decoders," IEEE Trans. Signal Processing, vol. 50, pp. 1051-1064, May. 2002.

[7] L. Collin, O. Berder, P. Rostaing, and G. Burel, "Optimal minimumdistance based precoder for MIMO spatial multiplexing systems," IEEE Trans. Signal Processing, vol. 52, pp. 617-627, Mar. 2004.

[8] D. P. Palomar, J. M. Cioffi, and M. A. Lagunas, "Joint Tx-Rx beamforming design for multicarrier MIMO channels: a unified framework for convex optimization," IEEE Trans. Signal Processing, vol. 51, pp. 2381-2401, Sept. 2003.

[9] Y. Jiang, J. Li, and W. W. Hager, "Uniform channel decomposition for MIMO communications," IEEE Trans. Signal Processing, vol. 53, pp. 4283-4294, Nov. 2005.

[10] L. Garca-Ordoez, D. P. Palomar, A. Pags-Zamora, and J. R. Fonollosa, "High-SNR analytical performance of spatial multiplexing MIMO systems with CSI," IEEE Trans. Signal Processing, vol. 55, no. 11, pp. 54475463, Nov. 2007.

[11] R. A. Horn and C. R. Johnson, Matrix Analysis. New York: Cambridge University Press, 1988.

[12] L. G. Ordonez, A. P. Zamora, and J. R. Fonollosa, "Diversity and multiplexing tradeoff of multiple beamforming in MIMO channels," in Proc. Intl. Symp. Inform. Theory, 2005, pp. 1808-1812.

[13] Z. Wang and G. B. Giannakis, "A simple and general parametrization quantifying performance in fading channels," IEEE Trans. Commun., vol. 51, no. 8, pp. 1389-1398, Aug. 2003.

[14] D. Tse and P. Vishwanath, Fundamentals of Wireless Communication. Cambridge University Press, 2005.

[15] K. Boulle and J. C. Belfiore, "Modulation schemes designed for the Rayleigh channel," in Proc. Conf. Inform. Sciences Systems 1992, Princeton, NJ, pp. 288-293.

[16] J. Boutrous and E. Viterbo, "Signal space diversity: a power and bandwidth efficient diversity technique for Rayleigh fading channel," IEEE Trans. Inform. Theory, vol. 44, pp. 1453-1467, July 1998.

[17] Md. Z. A. Khan and B. S. Rajan, "Single-symbol maximum-likelihood decodable linear STBCs," IEEE Trans. Inform. Theory, pp. 2062-2091, May 2006.

[18] A. T. James, "Distribution of matrix variates and latent roots derived from normal samples," Annals Mathematical Statistics, vol. 35, no. 2, pp. 475-501, June 1964.

[19] M. K. Simon and M.-S. Alouini, Digital Communication over Fading Channels. John Wiley \& Sons Inc., 2005. 